\title{
LITERATUR
}

\section{Die EU und ihre Nachbarn - alte Rezepte für neue Herausforderungen?}

\author{
Barbara Lippert*
}

Bücher, Aufsätze und Arbeitspapiere zur Europäischen Nachbarschaftspolitik (ENP) haben derzeit Konjunktur. ${ }^{1}$ So, wie viele Kommissionsbeamte und nota bene auch Europapolitiker, die sich in den letzten Jahren mit der Erweiterung befasst hatten, nun das Feld der Nachbarschaftspolitik bestellen, so richten auch viele Erweiterungsexperten unter den Politikwissenschaftlern an Universitäten und in den Thinktanks ihre Aufmerksamkeit auf die Nachbarn der EU. Im Mittelpunkt des Interesses stehen zumal jene, die erst seit der Großerweiterung 2004 unmittelbare Nachbarn der Union geworden sind. Anknüpfungspunkte bieten die Forschung zur ,Erweiterung', zur ,Europäisierung unter den Aspekten der Konditionalität und Compliance, zur ,Demokratisierung und Transformation" und nicht zuletzt zur ,Außen- und Sicherheitspolitik' der EU. Auch in Nachschlagewerken und Sammelbänden zur europäischen Integration ist das Stichwort Europäische Nachbarschaftspolitik unterdessen fest etabliert. ${ }^{2}$

\section{Pfadabhängige Nachbarschaftspolitik}

Die Geburtsstunde der Nachbarschaftspolitik wird unterschiedlich, zumeist aber auf 2003/
Esther Brimmer und Stefan Fröhlich (Hrsg.): The Strategic Implications of European Union Enlargement, Center for Transatlantic Relations, Johns Hopkins University: Washington D.C. 2005, ISBN 0-9753325-8-9; 412 Seiten, 25 \$.

Nicolas Hayoz, Leszek Jesień und Wim van Meurs (Hrsg.): Enlarged EU - Enlarged Neighbourhood. Perspectives of the European Neighbourhood Policy, [Interdisciplinary Studies on Central and Eastern Europe, Band 2], Peter Lang: Bern u.a. 2005, ISBN 3-03910-624-4; 392 Seiten, $69 \mathrm{SFR}$

Martin Koopmann und Christian Lequesne (Hrsg.): Partner oder Beitrittskandidaten? Die Nachbarschaftspolitik der Europäischen Union auf dem Prüfstand, [Denkart Europa. Schriften zur Europäischen Politik, Wirtschaft und Kultur, Band 2], Nomos Verlagsgesellschaft: Baden-Baden 2006, ISBN 3-8329-1943-0; 226 Seiten, $29 €$.

2004 datiert. Eine erste Vergewisserung über mögliche Konsequenzen der Erweiterung nach Osten und dem östlichen Mittelmeer nahm aber schon der Europäische Rat auf seiner Tagung in Madrid im Dezember 1995 vor. ${ }^{3}$ Einer

* Dr. Barbara Lippert, Stellvertretende Direktorin des Instituts für Europäische Politik, Berlin; Lehrbeauftragte an der Humboldt Universität zu Berlin.

1 Vgl. u.a. Barbara Lippert: Assoziierung plus Gesamteuropäische Aufgabenkonföderation: Plädoyer für eine selbstbewusste Nachbarschaftspolitik der EU, in: integration 2/2006, S. 149-157; Judith Kelley: New Wine in Old Wine Skins: Promoting Political Reforms through the New European Neighbourhood Policy, in: Journal of Common Market Studies 1/2006, S. 29-55 und Roland Dannreuther: Developing the Alternative to Enlargement: The European Neighbourhood Policy, in: European Foreign Affairs Review 2/2006, S. 183-201 sowie Andreas Marchetti: The European Neighbourhood Policy. Foreign Policy at the EU's periphery, ZEI discussion paper C158, 2006.

2 Vgl. z.B. Werner Weidenfeld/Wolfgang Wessels (Hrsg.): Europa von A-Z. Taschenbuch der europäischen Integration, Baden-Baden 2006 oder Wolfgang W. Mickel/Jan M. Bergmann (Hrsg.): Handlexikon der Europäischen Union, Baden-Baden 2005.

3 Vgl. Barbara Lippert: Die Union und ihre Nachbarn nach dem Verfassungsvertrag, in: Mathias Jopp/ Saskia Matl (Hrsg.): Der Vertrag über eine Verfassung für Europa. Analysen zur Konstitutionalisierung der EU, Baden-Baden 2005, S. 367-379, hier S. 368-369. 
der dort in den Schlussfolgerungen „Zur Vorbereitung auf das 21. Jahrhundert" genannten Nachbarn, die Türkei, hat unterdessen den Wechsel ins ,Lager' der Erweiterungspolitik vollzogen. Andere, wie die Ukraine, Belarus, Moldau und die Länder des südlichen Mittelmeers von Syrien bis Marokko, zählen zu den Nachbarländern, für die die Europäische Union schon Mitte der 1990er Jahre neue Formen der Anbindung sowie die Euro-mediterranen Assoziierungsabkommen beziehungsweise die Partnerschafts- und Kooperationsabkommen entwickelte und unter Bezug auf die von der Kommission seit 2003 vorgelegten Papiere weitere strategische Antworten suchte. Der ,Etagenwechsel' von der Nachbarschafts- zur Erweiterungspolitik, von Kooperation und Assoziierung zur Mitgliedschaft bildet einen Hauptuntersuchungsgegenstand und einen Brennpunkt der politischen Debatte über die Zukunft der EU und ihrer (neuen) Nachbarn. Die Nachbarschaftspolitik ist zwar zwischenzeitlich in der Kommission institutionell fest verankert: Kommissarin Ferrero-Waldner ist nicht nur für die Außenbeziehungen, sondern explizit für die Europäische Nachbarschaftspolitik zuständig. Dennoch ist die Nachbarschaftspolitik in ihren Zielsetzungen, ihrem Inhalt und ihrer Struktur sowie den Verfahren bei weitem nicht so klar konturiert und vertraglich abgestützt wie die Erweiterungspolitik. ${ }^{4}$ Zudem hat die Erweiterungspolitik im Zuge der Heranführungsstrategie für die Länder Ostmitteleuropas zahlreiche Innovationen erfahren, bei denen die Nachbarschaftspolitik heute vielfach Anleihen macht. Die Pfadabhängigkeit der Nachbarschafts- von der Erweiterungspolitik zeigt sich bei den Personen, Strukturen wie den Inhalten. Hier stellt sich sogleich die konzeptionelle wie praktische Frage nach den Möglichkeiten und Grenzen der Übertragbarkeit beziehungsweise der notwendigen Anpassung von Instrumenten und Verfahren der Erweiterungspolitik. Wie werden gleichgerichtete Maßnahmen in unterschiedlichen Ländern wirken? Wie viel des
Erfolges hängt von einer, europäischen Perspektive', sprich dem Zuckerbrot und der Peitsche des Beitritts zur EU, ab?

Mit diesem Themenspektrum befassen sich drei hier vorzustellende Neuerscheinungen, die aus internationalen Tagungs- und Kooperationsprojekten hervorgegangen sind. Das Buch „The strategic implications of European Enlargement", herausgegeben von der USamerikanischen Politikwissenschaftlerin Esther Brimmer und dem in Erlangen lehrenden Politikwissenschaftler Stefan Fröhlich, geht den Konsequenzen der Erweiterung für die Europäische Union als strategischer Akteur in den internationalen Beziehungen nach. Der von Martin Koopmann und Christian Lequesne herausgegebene Sammelband setzt sich explizit mit der Nachbarschaftspolitik auseinander, wie sie im engeren Sinne von der Europäischen Union verstanden wird. Beide Perspektiven, ,die erweiterte Union und die erweiterte Nachbarschaft", stehen im Zentrum der von Nicolas Hayoz, Leszek Jesień und Wim van Meurs herausgegebenen Aufsatzsammlung ,Enlarged EU - Enlarged neighbourhood".

Strategische Implikationen der EU-Erweiterung

Der Band von Esther Brimmer und Stefan Fröhlich ist in drei Teile gegliedert. Vier Beiträge befassen sich mit den strategischen Auswirkungen der Erweiterung auf die EU, fünf Beiträge mit der erweiterten EU und ihren unmittelbaren Nachbarn im Osten und Süden und sieben Beiträge, und hierin wird die transatlantische Komponente (explizit in den Aufsätzen von Esther Brimmer und Antonio Missiroli) besonders deutlich, mit der erweiterten EU und den internationalen Beziehungen, darunter die Themen globale Umweltpolitik (David Michel), die transatlantische Kooperation in der Entwicklungspolitik (Patrick M. Cronin/Muriel Asseraf) und Fragen

4 Vgl. z.B. Waldemar Hummer: Die Union und ihre Nachbarn - Nachbarschaftspolitik vor und nach dem Verfassungsvertrag, in: integration 3/2005, S. 233-245. 
der globalen Weltwirtschaft (Daniel Gros/Leonor Coutinho). Alle diese Beiträge sind von namhaften Autorinnen und Autoren - zumeist Politikwissenschaftlern - aus Europa und den Vereinigten Staaten verfasst. Michael Leigh, heute Generaldirektor für die Erweiterung, zuvor stellvertretender Generaldirektor Aussenbeziehungen mit Verantwortung für die Nachbarschaftspolitik in der Europäischen Kommission, der zu dem nämlichen Thema den Überblicksaufsatz verfasst hat, fällt ein wenig aus dem Rahmen. Leigh durchmisst die Entwicklung der Nachbarschaftspolitik und sieht deren Ursprünge eindeutig in der jüngsten Erweiterung und der in den 1990er Jahren intensiv betriebenen Heranführungspolitik. Er zeichnet die Stationen der Nachbarschaftspolitik, die als Politik für das „Wider Europe“5 begann, nach. Ihre Ziele sind Stabilitätsexport, Sicherheit, Verbesserung des Lebensstandards und der good governance in den Nachbarländern; sie basiert auf den Prinzipien der Differenzierung und der an Kriterien gebundenen Konditionalität. Er diskutiert auch angesichts der unverhohlenen Skepsis, mit der die Attraktivität der Nachbarschaftspolitik betrachtet wird, inwieweit die gewünschten Stabilisierungs- und Entwicklungsziele in den Nachbarschaftsländern ohne den Anreiz der Mitgliedschaft realisiert werden können. Die Ambivalenz der Haltung der EU gegenüber den Nachbarschaftsländern kommt auch in seiner Charakterisierung zum Ausdruck, dass die Nachbarschaftsländer mehr und mehr als „Partner“ und nicht als „Drittstaaten“ anzusehen seien. Die Trennschärfe zur Mitgliedschaft wird insoweit auch absichtlich verwischt.

\section{Russland „in der Klemme“}

Leigh behandelt Russland als einen Sonderfall. Russland wolle nicht als Objekt der Nachbarschaftspolitik betrachtet werden, sondern suche eine strategische Partnerschaft im
Rahmen einer Politik der vier Kooperationsräume mit der EU. Darin kommt eine Aversion der Regierung Putin (wie der Vorgängerregierungen) gegenüber den paternalistischen Zügen zum Ausdruck, die sowohl die Erweiterungs- als auch die Nachbarschaftspolitik der Union trägt. Das war und ist mit dem Anspruch Russlands, zumindest als regionale Großmacht zu gelten, nicht zu vereinbaren.

Timofei Bordachev sieht Russland zunehmend ,in der Klemme“ und am Ende seiner Europapolitik angelangt, die rein auf bilaterale Beziehungen $\mathrm{zu}$ Kernländern der $\mathrm{EU}$ setzt. Russland müsse sich entscheiden, ob es „Satellit oder Partner“ (S. 151) der EU werden wolle, wobei diese Entscheidung von den inneren Verhältnissen und Entwicklungen Russlands zwischen den Polen Demokratisierung/Europäisierung und Autoritatismus abhänge. Für die Rolle eines Partners der EU sieht Bordachev das „Modell Norwegens“ als einen Orientierungspunkt an. Bordachev interpretiert die Beziehungen zwischen EU und Russland seit den frühen 1990er Jahren als eine Geschichte divergierender Perzeptionen und Erwartungen. Wichtige Wegmarken waren die gemeinsamen Strategie der EU von 1999 und die Phase 2001/2002, in der praktische Konsequenzen der Erweiterungspolitik (Stichwort Kaliningrad) zu verarbeiten waren.

\section{Ukraine und Türkei - Stabilitätsexport der EU?}

Sehr kritisch bewertet Nicolae Idu die Rolle der EU gegenüber den Ländern in der ,Zwischenregion" (S. 171) Ukraine und Belarus. Die EU habe bislang ein sehr niedriges Profil und geringe Ambitionen gezeigt. Erst durch die orange Revolution habe sich die Ukraine gewissermaßen selbst auf die politische Agenda der EU gesetzt. Idu unterstreicht das besondere Interesse neuer Mitgliedstaaten, vor allen Dingen Polens und der baltischen Staaten, an einer östlichen Dimension der

5 Mitteilung der Kommission an den Rat und das Europäische Parlament - Größeres Europa - Nachbarschaft: Ein neuer Rahmen für die Beziehungen der EU zu ihren östlichen und südlichen Nachbarn, KOM (2003) 194 endg., Brüssel, 11.03.2003. 
EU-Außenbeziehungen einschließlich einer Mitgliedschaftsperspektive für Nachbarn wie die Ukraine. Die Erwartung an die EU sei eindeutig die eines Stimulus und Ankers für Demokratisierung und Modernisierung. In einen größeren Kontext stellt László Kiss diese Interessenlagen und Präferenzen neuer Mitgliedstaaten. Auch er sieht eine besondere Verantwortung der neuen ostmitteleuropäischen Mitgliedstaaten in der EU, die europäische Orientierung in den (süd)östlichen Nachbarländern der Union zu stärken. Aber er weist auch auf Differenzierungen unter den neuen EU-Ländern, etwa zwischen Polen und Ungarn, hin. Kiss versteht die Nachbarschaftspolitik als eine Herausforderung für die EU, zwischen einer Überdehnung durch Erweiterung und den möglichen negativen Effekten einer Ausgrenzung der Nachbarschaftsländer zu wählen. Das Inklusions-/Exklusions-Dilemma ist die zentrale Problematik der Beziehungen zwischen der EU und den Nachbarn. Kiss erläutert ferner die besonderen transatlantischen Interessen der neuen Mitgliedstaaten und weist die Schlussfolgerung zurück, dass der alte OstWest-Konflikt nun durch einen West-WestKonflikt abgelöst würde. Im Rückblick auf die EU-internen Divergenzen über den Militäreinsatz der USA im Irak warnt Kiss, dass die europäische Identitätsbildung mittels einer Gegnerschaft zu den Vereinigten Staaten nur auf Kosten der Einheit Europas zu haben sein werde.

$\mathrm{Zu}$ verweisen ist auch auf den überaus lesenswerten Beitrag von Henri J. Barkey und Anne-Marie le Gloannec über die strategischen Implikationen eines Beitritts der Türkei zur EU. Obwohl die Autoren insgesamt zu der Einschätzung gelangen, dass die Mitgliedschaft der Türkei einen zusätzlichen politischen und wirtschaftlichen Nutzen für die EU bringen kann, sehen sie doch, dass die Türkei aufgrund ihrer speziellen Interessen, die nicht zuletzt aus ihrer exponierten geopolitischen Lage resultieren, den politischen Zusammenhalt in der EU und den in vieler Hinsicht nur schwachen außenpolitischen und strategi- schen Acquis arg strapazieren würde. Sie setzen sich sehr abgewogen mit gängigen Einschätzungen auseinander, wie mit der Modellund Brückenfunktion der Türkei gegenüber dem asiatisch-muslimischen Raum und dem Argument des innen- und sicherheitspolitischen Stabilitätsexports. Sie diskutieren wie auch Lothar Rühle in seinem Aufsatz zum ,Greater Middle East` geopolitische Konstellationen, die sich im Zuge des Türkei-Beitritts verändern oder neu entstehen. Sie gehen sowohl auf die außen- als auch im engeren Sinne verteidigungs- und sicherheitspolitische Dimension, so die Kooperation mit der NATO und die Mitwirkung an gemeinsamen Aktionen der EU ein. Sie resümieren: „They only add a limited comparative advantage to EU capabilities in terms of diplomacy, because of their own entanglements which will have to be undone first" (S. 150). Am Ende des Beitrags zeichnen die Autoren einen Bogen der außenpolitischen Übereinstimmung zwischen den beiden Peripherieländern Großbritannien und Türkei, der auch auf einer starken transatlantischen Bindung gründet. Wenn man hier noch Polen mit ins Bild nimmt, so mag man nachdenklich werden, welche Aussichten dieses Dreieck wohl für eine künftige EU-Außenpolitik bringen wird. Der von Brimmer/Fröhlich sorgfältig edierte Sammelband bietet sich nicht nur für die unmittelbar an der Erweiterung und den Nachbarn sondern für alle an der (Neu-)Ausrichtung der Außen- und Sicherheitspolitik der EU Interessierten an. Ein Manko ist freilich, dass durchweg kaum auf die einschlägige auf Deutsch publizierte Literatur zum Themenfeld verwiesen wird.

\section{ENP - imperial und doch halbherzig?}

Enger im Zugriff auf die Thematik ist der von Nicolas Hayoz, Leszek Jesień und Wim van Meurs herausgegebene Sammelband. Die Herausgeber stellen in ihrer Einführung mehrfach heraus, dass sie sich kritisch mit der Nachbarschaftspolitik der Union auseinandersetzen wollen. Die Autorinnen und Autoren des Sammelbands können insoweit als ,Be- 
troffene ' gelten, als viele ihre Wurzeln in neuen EU-Mitgliedstaaten, in Kandidatenländern oder Ländern der Nachbarschaftspolitik haben. Das führt offenbar bei einigen zu einer sehr starken Identifikation mit dem Gegenstand und einer beherzt-normativen Argumentation, der man aber nicht immer folgen möchte. Zu Recht verweist Antoinette Primatarova darauf, dass die EU nicht mit Europa gleich zu setzen sei. Wertungen, wie die folgende „Europeanisation agenda designed for neighbours is an imperial approach, even if it should be restricted to the export of certain forms of political organization and governance" (S. 38) scheinen jedoch überzogen und werden auch in einem nachfolgenden Beitrag der Autoren Nicolas Hayoz/Franz Kehl/Stephan Kuster (S. 61) problematisiert.

Der Band beginnt mit einem Überblick über die Regionen der Nachbarschaft der EU. Neben dem bereits zitierten Rundumschlag von Primatarova setzen sich Hayoz/Kehl/Kuster mit Osteuropa als Gegenstand der östlichen Nachbarschaftspolitik auseinander und Wim van Meurs mit dem westlichen Balkan, einer Region, die aus Sicht der EU wegen ihrer bereits konzedierten europäischen Perspektive nicht unter Nachbarschaftspolitik firmiert. Es folgen dann in einem zweiten Abschnitt Berichte zu ausgewählten Ländern Osteuropas von Russland über die Ukraine und Belarus bis hin zu eher übergreifenden Betrachtungen aus der Perspektive Polens und Estlands. Hervorzuheben ist insbesondere der Beitrag von Jesień „Looking Eastwards“, in dem die Beziehungen der EU nach der Erweiterung zu den Ländern Ostmitteleuropas diskutiert werden. Wie viele andere Autoren greift Jesień die Fragestellung auf, inwieweit es Gemeinsamkeiten und Unterschiede zwischen der Erweiterungs- und der Nachbarschaftspolitik gibt. Seine Gesamteinschätzung ist eher skeptisch „It may be called , policy“ by its authors, it may be useful to call it this way by the authorising member states, but it does not seem to be a consistent entity, particularly in light of past enlargement policy. It is not enough to copy instruments of the enlargement and say they will not lead to further enlargement" (S. 242). Jesień bekräftigt seine Zweifel an einer bürokratisch-inkrementell entwickelten Politik, die ihrer strategischen Zielsetzung nicht gewiss ist. Der Band schließt mit Länderberichten aus Südosteuropa. Krassimir Nikolov behandelt die Nachbarschaftspolitik aus der Sicht Bulgariens. Andere Beiträge befassen sich mit der Entwicklung der Demokratie in Serbien oder in Bosnien-Herzegowina. Insgesamt ist der Band unausgewogener und teilweise auch nachlässiger ediert als die beiden anderen hier besprochenen Bände. Viele Texte behalten den Charakter von Konferenzund Arbeitspapieren bei.

\section{Partner oder Beitrittskandidaten?}

Eine größere Dichte und Geschlossenheit weist der von Martin Koopmann und Christian Lequesne herausgegebene Sammelband zur ENP auf, der in der neuen Reihe der ASKO Europa-Stiftung ,Denkart Europa Schriften zur europäischen Politik, Wirtschaft und Kultur" erschienen ist. In den elf Beiträgen gehen Politikwissenschaftler vornehmlich aus Deutschland, Frankreich und Polen der Grundfrage „Partner oder Beitrittskandidat?“ nach. Sie liefern problemorientierte Beiträge zur Nachbarschaft im Osten, den Beziehungen der EU zu den Ländern des Mittelmeerraums und zu Herausforderungen wie der künftigen Energiepolitik der Einwanderungssowie der Kohäsionspolitik der EU im Kontext der Nachbarschaftspolitik. Sie stellen damit das ,anspruchsvolle Politikkonzept“ (Koopmann, S. 17) der ENP auf den Prüfstand. Die Beiträge sind teils Englisch, teils Deutsch verfasst beziehungsweise aus dem Französischen übersetzt. Ein nützliches englisches Summary ist allen Beiträgen voran gestellt. Manche Autorinnen und Autoren geben Empfehlungen für die weitere Gestaltung der Nachbarschaftspolitik, nahezu alle Beiträge schließen mit einer klaren Einschätzung zu den aufgeworfenen Fragen. 


\section{Mehr GASP wagen!}

Die durchweg lesenswerten und gründlich erarbeiteten Beiträge legen die Schwächen des ENP-Konzepts und seiner gerade erst angelaufenen praktischen Durchführung dar. ${ }^{6} \mathrm{Zu}$ den Schwachpunkten gehört, dass die ENP die Dialektik „Beitritt/Nichtbeitritt“ beziehungsweise „Inklusion/Exklusion“ (siehe den Beitrag von Dov Lynch über die ENP und GASP, S. 147 bzw. S. 150) nicht auflöst, dass sie also hinsichtlich des zukünftigen Status der Nachbarn - Partner oder Beitrittskandidaten - ambivalent bleibt. Ein weiteres „Defizit“ ist, dass die ENP, wie Dorothée Schmid illustriert, den Mittelmeerländern zu viel anbietet. Denn diese haben auch mittelfristig keine realistischen Aussichten, etwa den Binnenmarktacquis zu übernehmen (S. 123). Andererseits bietet die ENP den osteuropäischen Ländern, wobei die Ukraine insbesondere nach der orangen Revolution im Mittelpunkt des Forschungsinteresses steht, aus Sicht der betreffenden Länder zu wenig. Für die Ukraine wird vielfach der Weg der Assoziierung als Zwischenschritt angesehen, wobei das politische und wirtschaftliche Potenzial der Ukraine auszuloten wäre, die nach ihrer Revolution in Orange einen Anspruch auf privilegierte Beziehungen zur EU und eine Beitrittsperspektive geltend macht. Andrea Gawrich sieht in der Beziehung zur Ukraine einen Lackmustest für das ENP-Konzept (S. 49). Nicht alle Autorinnen und Autoren teilen ihre Ansicht, dass die Erweiterungspolitik eine komplementäre Strategie zur Erweiterung (S. 51) sein solle. Lynch argumentiert überzeugend, dass die ENP auch konzeptionell sehr viel stärker als Außenpolitik verstanden und entwickelt werden müsse. Folgerichtig leidet auch die ENP an den Schwächen der GASP und ESVP insgesamt und legt den enormen Bedarf an Kohärenz zwischen den unterschiedlichen Instrumenten, über die die
EU in den Außenbeziehungen verfügt, bloß. Diesen Zusammenhang unterstreicht auch Steffen Angenendt. Er erwartet einen wichtigen Beitrag der Nachbarschaftspolitik zur Migrationspolitik der EU, die sich bislang auf dem kleinsten gemeinsamen Nenner bewegt und sich auf restriktive Positionen zur Grenzsicherung und Rückübernahmepolitik beschränkt. Angenendt begrüßt, dass die ENP einen neuen Anstoß gibt, um in der Einwanderungspolitik stärker außenpolitische Gesichtspunkte zu berücksichtigen und damit die migrationspolitischen Herausforderungen „Integration, Demografie, Konkordanzfähigkeit, Arbeitsmärkte, innere Sicherheit" (S. 196) strategisch und umfassend anzugehen. Die Länder der Nachbarschaftspolitik weisen ein hohes Migrationspotenzial auf, wobei Angenendt konstatiert, dass in erster Linie nicht ,die Armen, die Landbevölkerung, sondern die westlich orientierten, besser ausgebildeten städtischen Eliten, die über das nötige Kapital, die Kontakte und die Fähigkeiten verfügen, um sich auch in anderen Ländern ein Leben aufbauen zu können" (S. 191), aus Nordafrika und (Süd-)Osteuropa in die EU einwandern werden.

\section{Konkurrierende Konzepte?}

Schmid sieht konzeptionelle Defizite der ENP gegenüber den Mittelmeerländer, die ja über eine lange Tradition privilegierter Beziehungen zur EG/EU seit der globalen Mittelmeerpolitik der 1970-er Jahre verfügen. Sie rechnet mit Reibungen zwischen dem Konzept der ENP und dem der Euromediterranen Partnerschaft. Die Bilanz des Barcelona-Prozesses, der ja im Zentrum der Euromediterranen Partnerschaft steht, ist ernüchternd: ,die Europäische Union ist und bleibt Impulsgeber und Hauptakteur des Barcelona-Prozesses" (S. 116). Die ENP bietet auch nicht den Schlüssel zur Verbesserung der Beziehungen EU-Russland. Vielmehr erscheint die ENP als eine un-

6 Etwas aus dem Rahmen fällt der verwirrende Beitrag über, ,Territoriale Ungleichgewichte in Europa - Die Kohäsion auf dem Prüfstand der Erweiterung“ von Marie-Claude Maurel, der mit pauschalen Urteilen über die neuen Mitgliedstaaten aufwartet: „Die Beitrittsländer wollen weder ein mächtiges noch ein soziales Europa. Sie wollen sich nicht dem Modell eines Sozialstaats anschließen, den sie für reformunfähig halten“ (S. 224). 
geheure Herausforderung für Moskau. Anne de Tinguy sieht ähnlich wie Bordachev dass die russische Regierung sich, angestoßen durch die ENP, überlegen muss, inwieweit sie in Brüssel primär einen Konkurrenten im postsowjetischen Raum sieht oder zu einer partnerschaftlichen Beziehung gelangen will. De Tinguy zeichnet das Geflecht der Kooperation im Rahmen der vier gemeinsamen Räume nach, ein Ansatz, der mehr als eine Variante der ENP ist. Denn die Machtasymmetrie, die ansonsten die Beziehungen der EU zu den Nachbarn charakterisiert, ist so im Falle Russlands nicht gegeben. De Tinguy plädiert für ein neues, allerdings noch unbestimmtes Format für den Dialog EU-Russland, der auf den Gipfeltreffen wie jüngst im Mai 2006 eher symbolisch als substanziell ist. Das größte Hindernis für Fortschritte in den Beziehungen bleibt aus Sicht de Tinguys die Differenz im Hinblick auf die Werte und politische Ordnung. Auch als Gasgroßmacht ist Russland ein schwieriger Partner für die EU. In seinem Beitrag über Energienetze bietet Gilles Lepesant ein optimistisches Szenario an, in dem Energie als Integrationsfaktor wirken kann und ,durch die Institutionalisierung konkreter gemeinsamer Interessen Antagonismen und Verdächtigungen“ (S. 179) aufzulösen vermag.

Auch die USA kommt ins Spiel bei der Nachbarschaftspolitik. Genauer geht diesen Implikationen Carlo Masala in seinem Beitrag über die US-amerikanischen Perzeptionen der Mit- telmeerpolitik der EU nach. Er sieht ein potenzielles Spannungsfeld zwischen der Greater Middle East Initiative der Amerikaner und der ENP. Auswege könnte eine stärker auf funktionale Kooperation setzende Politik beider Seiten bieten, die entlang der Problemfelder Migration, Energiesicherheit, fundamentalistischer Terrorismus etc. verläuft. Das ließe Differenzen in den Hintergrund treten, die zwischen EU-Mitgliedstaaten und den USA hinsichtlich der Ordnungsfragen und Methoden des Interventionismus oder des, regime change" bestehen. Schließlich wird in einigen Beiträgen thematisiert, inwiefern im Zuge der Nachbarschaftspolitik Trennlinien verschärft werden (zum Beispiel Piotr Zalewski zu Schengen und Osteuropa), wobei hier viel Raum für die Ausgestaltung funktionsfähiger, aber „freundlicher“ Schengengrenzen gesehen wird.

Trotz der vielfach konstatierten Schwächen der ENP werden kaum echte Alternativen diskutiert. Insgesamt gewinnt man den Eindruck, dass die ENP ein ernst zu nehmendes Angebot der EU ist, das sicherlich fortentwickelt werden muss und das in jedem Falle den politischen Nerv getroffen hat: die Strukturierung und Neuordnung des Nachbarschaftsraums der EU ist nicht abgeschlossen und bleibt eine Herausforderung, der nicht nur mit alten Rezepten beizukommen ist. Insofern wird die Nachbarschaftspolitik ein Dauerthema bleiben. 